\title{
Archéopages
}

Archéopages

Archéologie et société

40 | 04-07/2014

Villages

\section{Les apports de la plate-forme Archéopuits}

Jean-Marc Fémenias, Christophe Tardy et Rémi Chantant

\section{CpenEdition}

Journals

Édition électronique

URL : https://journals.openedition.org/archeopages/651

DOI : 10.4000/archeopages.651

ISSN : 2269-9872

\section{Éditeur}

INRAP - Institut national de recherches archéologiques préventives

\section{Édition imprimée}

Date de publication : 1 juin 2015

Pagination : 162-163

ISSN : 1622-8545

\section{Référence électronique}

Jean-Marc Fémenias, Christophe Tardy et Rémi Chantant, « Les apports de la plate-forme

Archéopuits », Archéopages [En ligne], 40 | 04-07/2014, mis en ligne le 01 juillet 2016, consulté le 23 janvier 2022. URL : http://journals.openedition.org/archeopages/651 ; DOI : https://doi.org/10.4000/ archeopages.651 
1- Barzan, Moulin du Fâ, Univ. Bordeaux 3 (17)

2- Lançon-de-Provence, Constantine, F. Verdun (13)

3- Villetelle, Ambrussum, J.-L. Fiches (34)

4- Jonzac, Villa gallo-romaine, K. Robin (17)

5- Chateaumeillant, Oppidum de Mediolanum, S. Krausz (18)

6- Valros, Rec de Ligno, J.-B. Chevance (34)

7- Béziers, Le Gasquinoy, L. Buffat (34)

8- Carcassonne, Parking Gambetta, A. Bergeret (11)

9- Nîmes, Parking Jean Jaurès, J.-Y. Breuil (30)

10- Tourbes, Montferrier, M. Compan (34)

11- Périgueux, Camp de César, A. Colin (24)

12-Vieille Toulouse, Chemin de la Planho, P. Gardes (31)

13- Saintes, Clinique Richelieu, P. Poirier (17)

14- Saintes, Montlouis, J.-P. Baigl (17)

15- Bordeaux, L'Auditorium, K. Chuniaud (33)

16- Poussan, Roumèges, R. Bourgaut (34)

17- La Rochelle, L'Houmeau, T. Cornec (17)

18- Montbazin, La Reille, R. Bourgaut (34)

19- Sauvian, La Lesse, H. Pomarèdes (34)

20- Pons, Rue Franklin Roosevelt, S. Redais (17)

21- Villeneuve sur Lot, La Dardenne, A. Alcantara (47)

22- Saint Gence, La Gagnerie, C. Maniquet (87)

23- Naves, Tintignac, C. Maniquet (19)

24- Béziers, lieu-dit « Mazeran », R. Haurillon (34)

25- Béziers, La Courondelle, E. Gomez (34)

26- Carcassonne, Lo Badarel, M. Guillaume (11)

27- Montluçon, Les Hauts de Buffon, U. Cabezuelo (03)

28- Rezé, Chapelle Saint Lupien, O. De Peretti (44)

29- Saintes, allée de La Poudrière, P. Poirier (17)

$\Phi$

(1) Chantiers Inrap

(1)

Chantiers Collectivités locales

(1) Chantiers CNRS / Universités / Associations

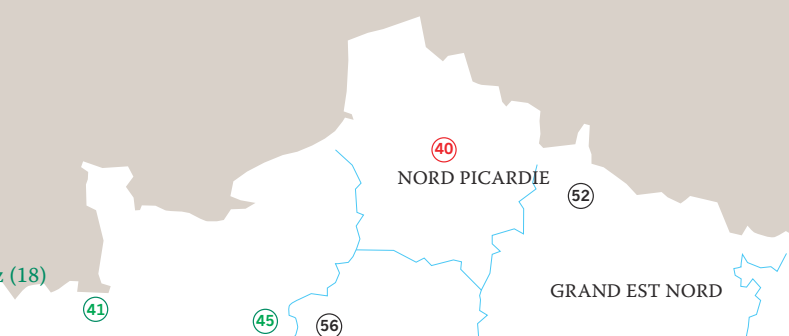

(45) (56)

CENTRE ÎLE-DE-FRANCE
(50)

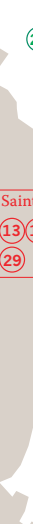

(28)

Sain
39

(30) GRAND EST SUD

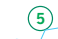

5

(17) (22)

(27)

RHÔNE ALPES AUVERGNE 31- Claira, Saint Jaume de Crest, V. Canut (66)

- Nîmes, Mas de Vignolles 14, H. Pomarèdes (30)

33- Apcher, Le Château, I. Rémy (48)

34- Istres, Le Castellan, F. Marty (13)

35- Nîmes, Zac des Carmes, O. Maufras (30)

36- Lodève, Capitaine Moran, A. Bergeret (34)

37- Périgueux, Bd Lakanal, W. Migeon (24)

38- Béziers, Zac de Mazeran, R. Haurillon (34)

39- Entrains-sur-Nohain, Rte d'Étais, G. Vincent (58) 40- Nesle / Mesnil-Saint-Nicaise, A. Rousseau (80)

41- Créhen, Château du Guildo, L. Beuchet (22)

42- Jublains, Domus, A. Bocquet (53)

43- Pisany, La Garenne, L. Maurel (17)

44- Éauze, La Cieutat, P. Pisani (32)

45- Vieil-Évreux, Gisacum, S. Bertaudière (27) 46- Istres, Forum des Carmes, F. Paone (13)

47- Andilly, 17 rue Saint-Nicolas, S. Vacher (17)

48- Castries, Mas du Roux, H. Pomaredes (34)

49- Aimargues, Saint Gilles le Vieux, O. Maufras (34)

50- Ossé, La Claraiserie, J. Le Gall (35)

51- Toulon, Ilot Baudin, N. Molina (83)

52- Charleville-Mézières, Le Clos Paul, J. Marian (08)

53- Magalas, Monfo-Magalas, O. Ginouvez (34)

54- Perpignan, 10 rue de l'Académie, I. Remy (66)

55- Entrains sur Nohain, Voie Romaine, S. Venault (58)

56- Chartres, 6 ruelle du Grand Sentier, J. Viret (28)

57- Mauguio, Lallemand, L. Tarrou (34)

58- Mudaison, Les Aubettes, Y. Pascal (34)

59- Saint-Rémy de Provence, Marceau, E. Sagetat (13)

Les apports de la plate-forme Archéopuits

Jean-Marc Féménias Archéopuits

Christophe Tardy Inrap

Rémi Chantant Inrap

Les moyens utilisés jusqu'à la fin des années 1990 ne pouvaient assurer les garanties suffisantes de sécurité dans les actions de descente dans les excavations, de remontée des déblais issus du remplissage et d'évacuation des eaux de pompage. Lors des campagnes de fouille entamées en 1999 au Moulin du Fâ (Charente-Maritime), sous la direction d'Alain Bouet, JeanMarc Féménias, archéologue amateur et marin-pompier, attire l'attention sur l'impérieuse nécessité d'établir des règles propres à la recherche archéologique dans les puits. Un prototype de plate-forme en tubes d'échafaudage est élaboré en z00‡ sur la fouille de Constantine (Bouches-duRhône). I'investissement est suffisamment important pour motiver la création de l'association Archéopuits et la mise à disponibilité d'une personne en permanence au fonctionnement de celle-ci. Ce dispositif est utilisé ensuite lors de nombreuses fouilles programmées sur des sites comprenant des puits [ill. 1], ce qui a permis de démontrer le potentiel scientifique de ce type de structure, de revoir l'interprétation de certains sites (comme celui de la petite agglomération routière gallo-romaine d'Ambrussum [Fiches, 2012]), de perfectionner les procédures, en relation avec les règles de sécurité strictes appliquées en archéologie préventive [ill. 2], et le protocole de la fouille manuelle des puits et des plateaux techniques afférents. Sur les chantiers archéologiques de l'A75 (Fabre et al., 2009), entre Béziers et Pézenas, et du parking Jean-Jaurès à Nimes (Breuil et al., 2014), a été systématiquement mis en place un protocole de tamisage des sédiments sur site, dès la sortie du puits, ainsi que le traitement rapide des mobiliers fragiles et gorgés d'eau qui en étaient extraits. Par voie de conséquence, c'est tout un cortège d'études spécialisées qui a découlé des nombreuses découvertes, réalisées en lien avec le grand intérêt chrono stratigraphique des comblements issus de ces ensembles clos et profonds et la qualité souvent inégalée de préservation de ses mobiliers en contexte humide. Si tous les puits ne sauraient être fouillés au moyen de cette méthode, l'utilisation de la plate-forme se justifie particulièrement dans certaines fouilles, notamment dans des contextes géologiques particuliers, avec des contraintes spatiales, des problèmes d'accessibilité aux engins mécaniques ou des enjeux de sécurité dus à la proximité de réseaux divers.

Le protocole de fouille permis par l'usage de cette plate-forme amène, selon nous, à relever la structure et à appréhender son remplissage avec une précision d'observation bien plus importante et efficace qu'avec la fouille à la pelle mécanique ; notamment en ce qui concerne l'exploration du conduit du puits, sa description et la compréhension de sa construction, le relevé topographique des mobiliers archéologiques et des études stratigraphiques fines, indispensables aux études paléoenvironnementales et, enfin, le prélèvement optimal des mobiliers fragiles. 
CHUTE DU

PERSONNEL

FEU D'ORIGINE ÉLECTRIQUE

BLESSURES

INSTABILITÉ

RISQUE

ÉLECTRIQUE

USURE

ÉBOULEMENT

ÉCLATEMENT

PERTE DE

CONNAISSANCE

CHUTE D'OBJET

ASPHYXIE

INONDATION

AFFAISSEMENT

RISOUES

MICROBIENS

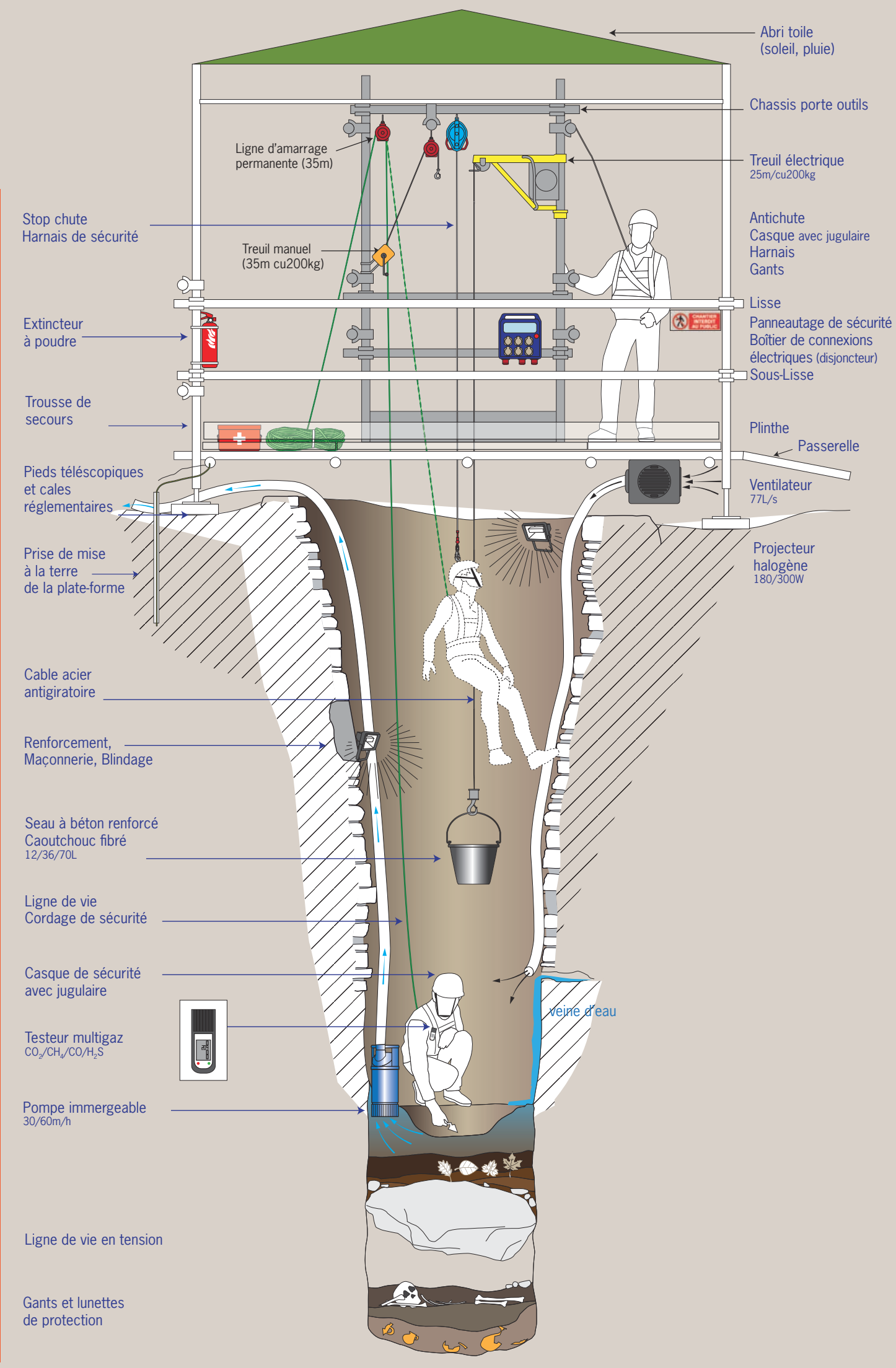

ณ

点 